\title{
The DNA Framework of Visualization
}

\author{
Yuri Engelhardt ${ }^{1(\otimes)}\left(\mathbb{D}\right.$ and Clive Richards ${ }^{2}$ (D) \\ 1 University of Twente, Enschede, The Netherlands \\ yuri.engelhardt@utwente.nl \\ 2 Birmingham City University, Birmingham, UK \\ clive.j.richards@me.com
}

\begin{abstract}
A comprehensive framework is presented for analyzing and specifying an extensive range of visualizations in terms of their fundamental 'DNA' building blocks of visual encoding and (de)composition.
\end{abstract}

\section{Introduction}

In their "Tour through the Visualization Zoo", Heer et al. (2010) say that "all visualizations share a common 'DNA' - a set of mappings between data properties and visual attributes" (p. 60). We use this metaphorical idea of the 'DNA of visualization' in a similar vein, taking it to the extent of identifying a comprehensive set of individual DNA building blocks of visualizations and the rules for combining them. This allows for the construction of a broad range of different types of visualizations.

Numerous authors have written about analysing visualizations and various visualization grammars have been developed (e.g. Vega-Lite www.vega.github.io). We have reviewed this work and we have identified gaps in what is covered (see Engelhardt and Richards 2018). The framework we present here fills these gaps. It:

1. provides a comprehensive system for exploring and checking design possibilities for visualization.

2. offers a system of tree diagrams for representing (de)composition and visual encoding in visualizations (constructed from their 'DNA').

3. presents a way of describing visualizations with rigorously systematic natural language sentences, which specify (de)composition and visual encoding.

4. covers a very broad design space of visualization, not only including visual representations that involve numerical information, but also visualizations such as family trees, Venn diagrams, flow charts, texts using indenting, technical drawings and scientific illustrations.

The above characteristics of the framework enable the analysis and comparison of visualization types, and potentially provide a design method for exploring visualization options. Like academic work in linguistics, the work presented here is primarily not prescriptive but descriptive, in the sense that it enables the understanding and modelling of (graphic) language.

Y. Engelhardt and C. Richards—-both authors contributed equally to the work

(C) The Author(s) 2020

A.-V. Pietarinen et al. (Eds.): Diagrams 2020, LNAI 12169, pp. 534-538, 2020.

https://doi.org/10.1007/978-3-030-54249-8_51 


\section{The Building Blocks and How They Relate to Each Other}

The process diagram in Fig. 1 shows all our DNA building blocks and their possible relationships for expressing information visually. For ease of use we have given each DNA building block a three letter abbreviation. The DNA building blocks fall into several main groups: types of information to be represented, visual encodings to represent them, visual components that make up the visualization, and any directions or layout principles that may be involved. Visual encodings can be used for arranging, varying or linking visual components. Arranging visual components into meaningful configurations is how visualizations are constructed.

Our visual encodings include the use of Bertin's 'visual variables', some Gestalt principles of perception (e.g. grouping by proximity) and other fundamental ways of expressing information visually. A visual component can be involved in several different visual encodings, simultaneously representing different types of information.

We refer to a 'well-formed' combination of DNA building blocks as a visualization pattern. Many common visualization patterns have been given a name (e.g. 'pie chart') and are generally referred to as 'chart types', while novel or rare patterns often do not have a name (yet). A visualization pattern can be transformed into another pattern by adding, replacing or removing one or more DNA building blocks. A large number of patterns has been analyzed using this system. Some examples can be found in Fig. 2. Many more analyses are on our accompanying website: www.VisDNA.com.

\section{Discussion and Conclusions}

The framework offers a potential research tool for exploring various kinds of commonalities, family resemblances and differences between visualization patterns within collections of graphic representations. The DNA building blocks and the precisely defined methods by which they can be combined (see www.VisDNA.com) offer the potential for machine readable specifications. This may serve as a basis for a system providing computer generated visualization advice, which could be linked to a rendering engine in order to produce actual visualizations and variants of them.

Because of its flexible building block structure, additional DNA elements may be added to the framework to accommodate any new constructions that one may want to describe and that cannot be fully analysed using the current scheme. An example may be the addition of DNA building blocks for interactivity in visualizations. 


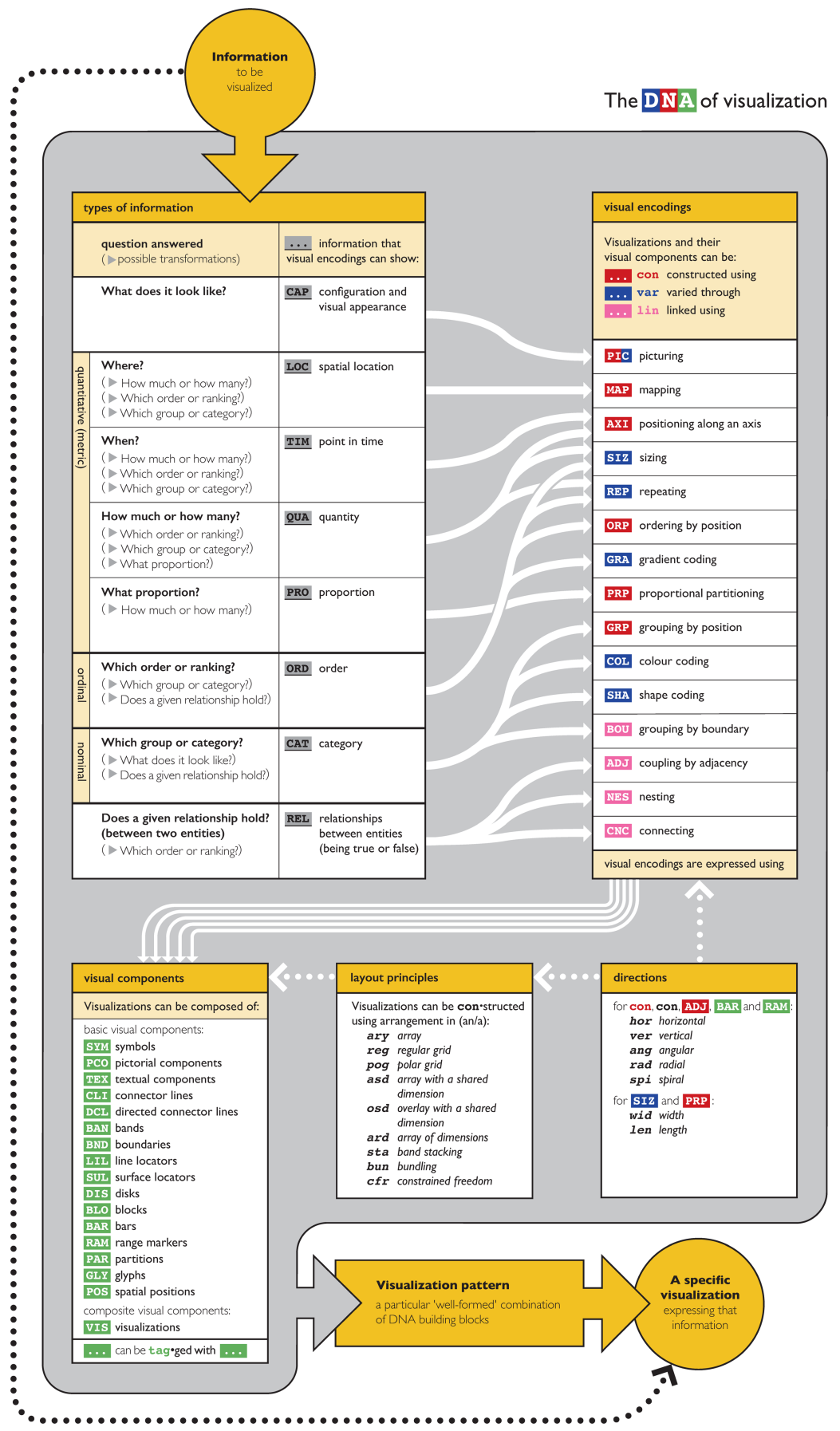

Fig. 1. Process diagram showing our DNA building blocks and their possible relationships. 


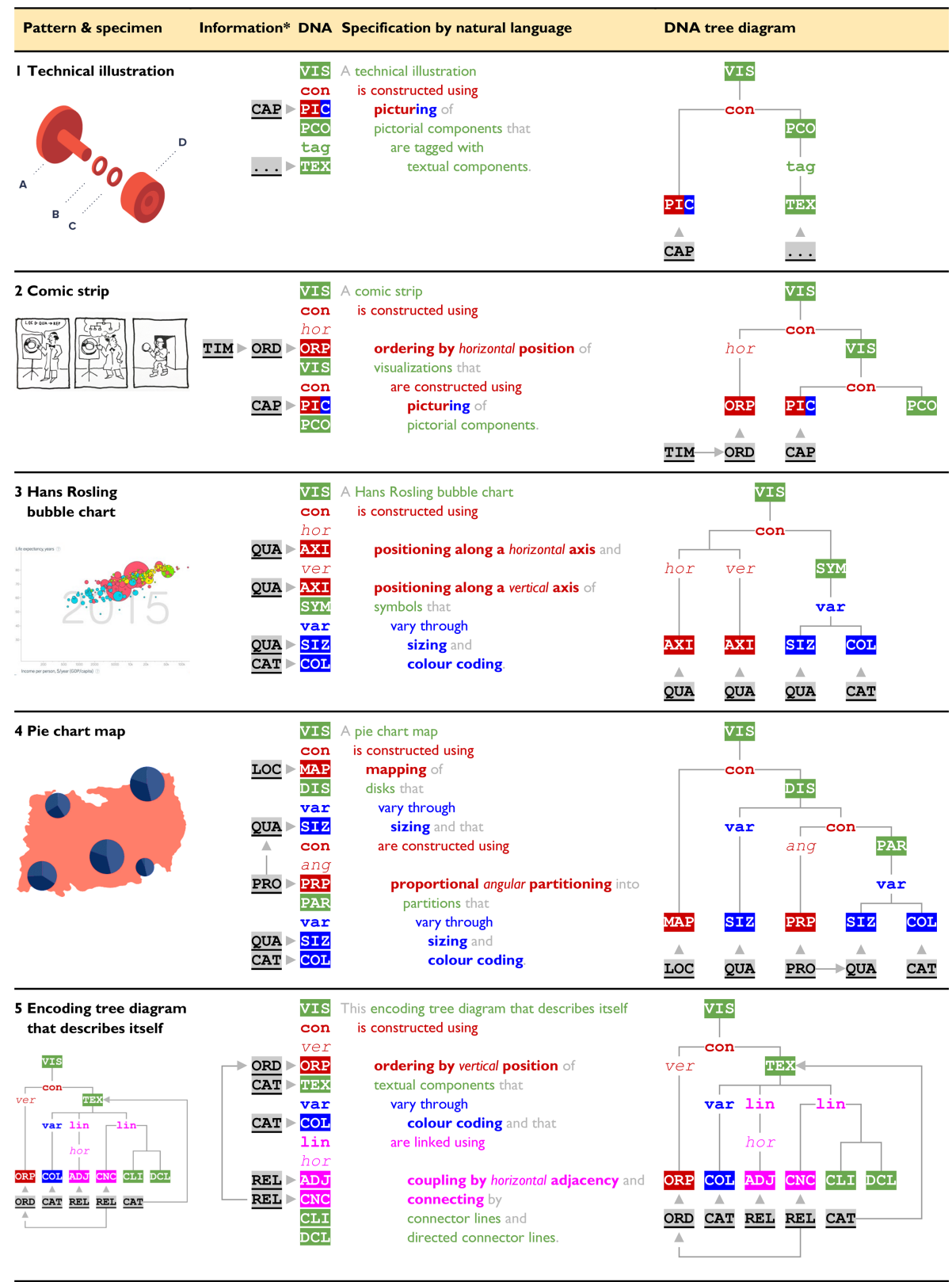

* TIM Point in time LOC Location QUA Quantity PRO Proportion ORD Order CAT Category CAP Configuration and appearance REL Relationships ... Placeholder for any type of information

Transformations between these types of information are shown as grey arrows.

Fig. 2. Example DNA analyses. See www.VisDNA.com for analyses of many more types of visualizations. Images at 1 and 4 courtesy of the DataVizProject by Ferdio, Creative Commons Attribution-NonCommercial-NoDerivatives 4.0 International License: www.datavizproject.com 


\section{References}

Engelhardt, Y., Richards, C.: A framework for analyzing and designing diagrams and graphics. In: Chapman, P., Stapleton, G., Moktefi, A., Perez-Kriz, S., Bellucci, F. (eds.) Diagrams 2018. LNCS (LNAI), vol. 10871, pp. 201-209. Springer, Cham (2018). https://doi.org/10.1007/9783-319-91376-6_20

Heer, J., Bostock, M., Ogievetsky, V.: A tour through the visualization zoo. Commun. ACM 53(6), 59-67 (2010). https://doi.org/10.1145/1743546.1743567

Open Access This chapter is licensed under the terms of the Creative Commons Attribution 4.0 International License (http://creativecommons.org/licenses/by/4.0/), which permits use, sharing, adaptation, distribution and reproduction in any medium or format, as long as you give appropriate credit to the original author(s) and the source, provide a link to the Creative Commons license and indicate if changes were made.

The images or other third party material in this chapter are included in the chapter's Creative Commons license, unless indicated otherwise in a credit line to the material. If material is not included in the chapter's Creative Commons license and your intended use is not permitted by statutory regulation or exceeds the permitted use, you will need to obtain permission directly from the copyright holder.

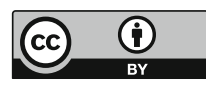

